\title{
Surface magnetic fields in pulsars
}

\section{George I. Melikidze and Janusz Gil}

Institute of Astronomy, University of Zielona Gora, Lubuska 2, 65-265, Zielona Gora, Poland email: gogi@astro.ia.uz.zgora.pl, jag@astro.ia.uz.zgora.pl

\begin{abstract}
Observations of hot-spot thermal X-ray emission from radio pulsars implicate that surface magnetic field (SMF) at the polar cap is much stronger than the conventional dipolar component estimated from the pulsar spin-down. This strongly suggests that SMF is dominated by the crust anchored small scale magnetic field. We present the observed values of black body temperature and bolometric luminosity of X-ray emission from hot polar caps of a number of pulsars. In all cases the inferred value of SMF is close to $10^{14} \mathrm{G}$.
\end{abstract}

Keywords. Stars: neutron - stars: magnetic field - pulsars: PSR J0108-1431

Thermal X-ray emission seems to be a quite common feature of radio pulsars. XMM Newton and Chandra observations demonstrated that not only relatively young and hot pulsars could be seen in thermal X-rays. In older and cooler pulsars this radiation can originate on the surface of hot polar caps Pavlov et al. (2008). Therefore, temperature and bolometric luminosity of black body radiation from hot spots allow us to shed some light on the main features of the polar cap region in radio pulsars. The most unexpected result was estimation of the hot spot surface area (derived from the spectral fit with a hot-spot black body model), which appears to be much smaller than the conventional polar cap surface area $A_{0}(P) \simeq 6.6 \times 10^{8} P^{-1}\left[\mathrm{~cm}^{2}\right]$. Here $P$ is the pulsar period. The only natural explanation of this observational feature is an assumption that the magnetic field at the stellar surface differs essentially from the pure dipole field. The standard model for pulsars assumes that the source of the pulsar activity is associated with the inner acceleration region (called gap) above the polar cap, where the electric field has a component along the magnetic field lines. Particles (electrons and positrons) are accelerated in both directions: outwards and towards the stellar surface. Consequently, outstreaming particles generate the magnetospheric X-ray emission while the backstreaming particles heat the surface and provide necessary energy for the thermal emission from hot polar cap.

The black body fit allow us to obtain directly the temperature $T_{\mathrm{BB}}$ and the surface area of the hot spot. As mentioned above, in most cases $A_{\mathrm{BB}}$ is much less than $A_{0}$ (Zhang et al. (2005); Kargaltsevet al. (2006)) and it can be easily explained by assuming that the surface magnetic field of pulsars differs significantly from the pure dipole one. One can estimate an actual surface magnetic field by the magnetic flux conservation law as $b=A_{0} / A_{\mathrm{BB}}=B_{s} / B_{d}$. Here $B_{d} \simeq 2 \times 10^{12}\left(P \dot{P}_{-15}\right)^{0.5}[\mathrm{G}]$ is a dipolar magnetic field and $P \dot{P}_{-15}$ is a period derivative. Then $B_{s} \simeq 1.3 \times 10^{21} A_{\mathrm{BB}}^{-1}\left(P \dot{P}_{-15}\right)^{0.5} \mathrm{G}$. In most cases $b \gg 1$ implying $B_{s} \gg B_{d}$, and $T_{\mathrm{BB}} \simeq(2 \div 4) \times 10^{6} \mathrm{~K}$ (see figure 1$)$.

For such a scenario Gil, Melikidze \& Geppert (2007) have proposed the model for the inner acceleration region, which assumes that the gap operates in such a way that surface temperature is always very close the certain, unambiguously defined by the surface magnetic field critical temperature $T_{i}$ above which iron ions are thermally ejected from the surface up to the maximum co-rotational limited amount. This model is known as the Partially Screened Gap (PSG) model, as it assumes that the potential drop near the stellar surface is partially screened by the positive ions. The screening factor $\eta$ can be defined as $\eta=1-\rho_{i} / \rho_{\mathrm{GJ}}=1-\exp \left(C_{i}-\varepsilon_{c} / k T_{s}\right)$. Here $\rho_{i}$ and $\rho_{\mathrm{GJ}}$ are the actual and 

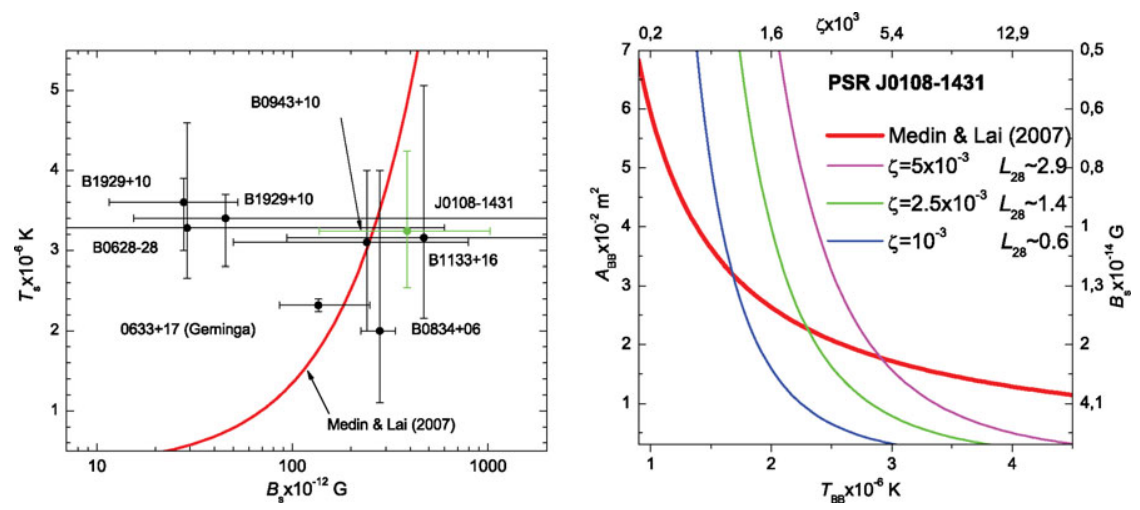

Figure 1. (Left panel) Positions of a number pulsars (with the known BB spectra fitting parameters) on the $T_{\mathrm{BB}}-B_{s}$ diagram. The thick red line represents the Medin \& Lai dependence $T_{i}=T i\left(B_{s}\right)$ for $C_{i}=30$. Recently observed very old PSR J0108-1431 is marked by a green color. (Right panel) Lines of the constant bolometric luminosity for PSR J0108-1431.

co-rotational charge densities, $\varepsilon_{c}$ is the cohesive energy of ions, $T_{s}$ is the surface temperature and the parameter $C_{i} \approx 30$ depends on magnetic field strength and temperature. Then we can define the critical temperature as a temperature when $\rho_{i}=\rho_{\mathrm{GJ}}$. Thus $T_{i}=\varepsilon_{c} / C_{i} k$.

In order to estimate $T_{i}$ we need to know the cohesive properties of the surface matter. This problem has been recently examined by Medin \& Lai (2007). They have estimated the critical temperature of the neutron star's iron crust and concluded that if the surface magnetic field is about $10^{14}$ Gauss, then the gap can be formed even if the surface temperature reaches few million $\mathrm{K}$, i.e., the condition $\rho_{i}<\rho_{\mathrm{GJ}}$ is satisfied even for such a high temperature. The dependence of $T_{i}=T_{i}\left(B_{s}\right)$ obtained by Medin \& Lai (2007) is shown as red lines in both panels of figure 1.

Let us emphasize once more that within PSG model the hot spot temperature should be very near to the critical temperature $T_{\mathrm{BB}} \simeq T_{i}$. Therefore, while fitting the hot spot $\mathrm{BB}$ spectra $A_{\mathrm{BB}}$ and $T_{\mathrm{BB}}$ should not be treated as an independent parameters. The pulsars, which X-ray spectra can be fitted to the black body emission from the hot spot should obey the $T_{i}=T_{i}\left(B_{s}\right)$ dependence. Assuming $T_{i}=T_{\mathrm{BB}}$, we can rewrite the function $T_{i}=T_{i}\left(B_{s}\right)$ and obtain for a given pulsar

$$
A_{\mathrm{BB}}=F\left(P, \dot{P}_{-15}, T_{\mathrm{BB}}\right) \text {. }
$$

Using PSG model we can also estimate the X-ray luminosity $L_{X} \approx 4 A_{\mathrm{BB}} \sigma T_{\mathrm{BB}}^{4}$, and its efficiency as $\zeta=L_{X} / L_{\mathrm{SD}}$, where $L_{\mathrm{SD}}=3.9 \times 10^{31} \dot{P}_{-15} P^{-3}\left[\mathrm{erg} \mathrm{s}^{-1}\right]$ is the pulsar spindown energy loss rate. The lines of the constant efficiency for PSR J1702-19 are shown in figure 1 (right panel).

\section{Acknowledgements}

The work was supported by Grants NN203 273833 (JG) and GNSF ST06/4-096 (GM).

\section{References}

Gil, J., Melikidze, G. I., \& Geppert, U. 2003, $A \& A$ 407, 315

Kargaltsev, O., Pavlov, G. G., \& Garmire, G. P. 2006, ApJ 636, 406

Medin, Z., Lai, D. 2007, MNRAS 382, 1833

Pavlov, G. G., Kargaltsev, O., Wong, J. A., \& Garmire, G. P. 2008, astro-ph/0803.0761

Zhang, B., Sanwal, D., \& Pavlov, G. G. 2005, ApJ 624, L109 